\title{
Water-induced $\mathrm{MAPbBr}_{3} @ \mathrm{PbBr}(\mathrm{OH})$ with enhanced luminescence and stability
}

\author{
Kai-Kai Liu', Qian Liu', Dong-Wen Yang ', Ya-Chuan Liang ${ }^{1}$, Lai-Zhi Sui', Jian-Yong Wei', Guo-Wei Xue', Wen-Bo Zhao ', \\ Xue-Ying Wu', Lin Dong (1) and Chong-Xin Shan ${ }^{1}$
}

\begin{abstract}
Poor stability has long been one of the key issues that hinder the practical applications of lead-based halide perovskites. In this paper, the photoluminescence (PL) quantum yield (QY) of bromide-based perovskites can be increased from $2.5 \%$ to $71.54 \%$ by introducing water, and the PL QY of a sample in aqueous solution decreases minimally over 1 year. The enhanced stability and PL QY can be attributed to the water-induced methylamino lead bromide perovskite $\left(\mathrm{MAPbBr}_{3}\right) @ \mathrm{PbBr}(\mathrm{OH})$. We note that this strategy is universal to $\mathrm{MAPbBr}_{3}$, formamidine lead bromide perovskite $\left(\mathrm{FAPbBr}_{3}\right)$, inorganic lead bromide perovskite $\left(\mathrm{Cs}_{\mathrm{PbBr}}\right)$, etc. Light-emitting devices (LEDs) are fabricated by using the as-prepared perovskite as phosphors on a $365 \mathrm{~nm}$ UV chip. The luminance intensity of the LED is $9549 \mathrm{~cd} / \mathrm{m}^{2}$ when the driven current is $200 \mathrm{~mA}$, and blemishes on the surface of glass are clearly observed under the illumination of the LEDs. This work provides a new strategy for highly stable and efficient perovskites.
\end{abstract}

\section{Introduction}

In recent years, lead halide perovskites (LHPs) $\mathrm{APbX}_{3}$ $\left(\mathrm{A}=\mathrm{CH}_{3} \mathrm{NH}_{3}{ }^{+} / \mathrm{CH}\left(\mathrm{NH}_{2}\right)_{2}{ }^{+} / \mathrm{Cs}^{+}, \mathrm{X}=\mathrm{Cl}^{-} / \mathrm{Br}^{-} / \mathrm{I}^{-}\right)$have emerged as promising materials for photovoltaics and light-emitting diodes due to their attractive optical and electrical properties, such as high photoluminescence (PL) quantum yield $(\mathrm{QY})$, narrow emission spectrum, tuneable emission wavelength, high absorption coefficient, and long carrier diffusion length ${ }^{1-11}$. Profound developments have been witnessed in the fields of solar cells ${ }^{12-15}$, solidstate light-emitting diodes ${ }^{11,16-20}$, photodetectors ${ }^{21-23}$, and lasers ${ }^{7,24,25}$. However, the poor stability of LHPs, especially in water and polar solvents, remains a crucial issue that hampers their applications.

The origin of the instability of LHPs is generally attributed to their low formation energy, which makes

\footnotetext{
Correspondence: Lin Dong (Idong@zzu.edu.cn) or Chong-Xin Shan (cxshan@zzu.edu.cn)

${ }^{1}$ Henan Key Laboratory of Diamond Optoelectronic Materials and Devices, Key Laboratory of Material Physics, Ministry of Education, School of Physics and Microelectronics, Zhengzhou University, Zhengzhou 450052, China

${ }^{2}$ State Key Laboratory of Molecular Reaction Dynamics, Dalian Institute of Chemical Physics, Chinese Academy of Sciences, 457 Zhongshan Road, Dalian 116023, China
}

these materials vulnerable to light, heat, oxygen, and moisture, especially when water is involved ${ }^{26-29}$. In addition, the ions of LHPs with discrete components are exchanged with each other quickly, which results in severe shifts in the emissions. Strategies for improving the stability of LHPs can be classified into three types: compositional engineering, surface engineering, and matrix encapsulation ${ }^{30}$. The all-inorganic lead perovskite $\left(\mathrm{CsPBBr}_{3}\right)$, where methylamino (MA) ions are replaced with $\mathrm{Cs}$ ions, shows much higher stability, including thermal and environmental stability, than organic-inorganic perovskite ${ }^{31}$. Although the thermal and environmental stability of this perovskite has been improved through compositional engineering, poor moisture stability is still a serious problem. The stability of LHPs improves greatly when they are covered with long-chain surface agents, which has been widely demonstrated by researchers. However, LHPs usually lose surface ligands and then reunite and lose their colloidal stability during purification ${ }^{32}$. Matrix encapsulation has been applied to effectively enhance the stability and PL efficacy of perovskite. For example, Jia et al. prepared $\mathrm{CsPbX} / \mathrm{Cs}_{4} \mathrm{PbX}_{6}$ core/shell perovskite 


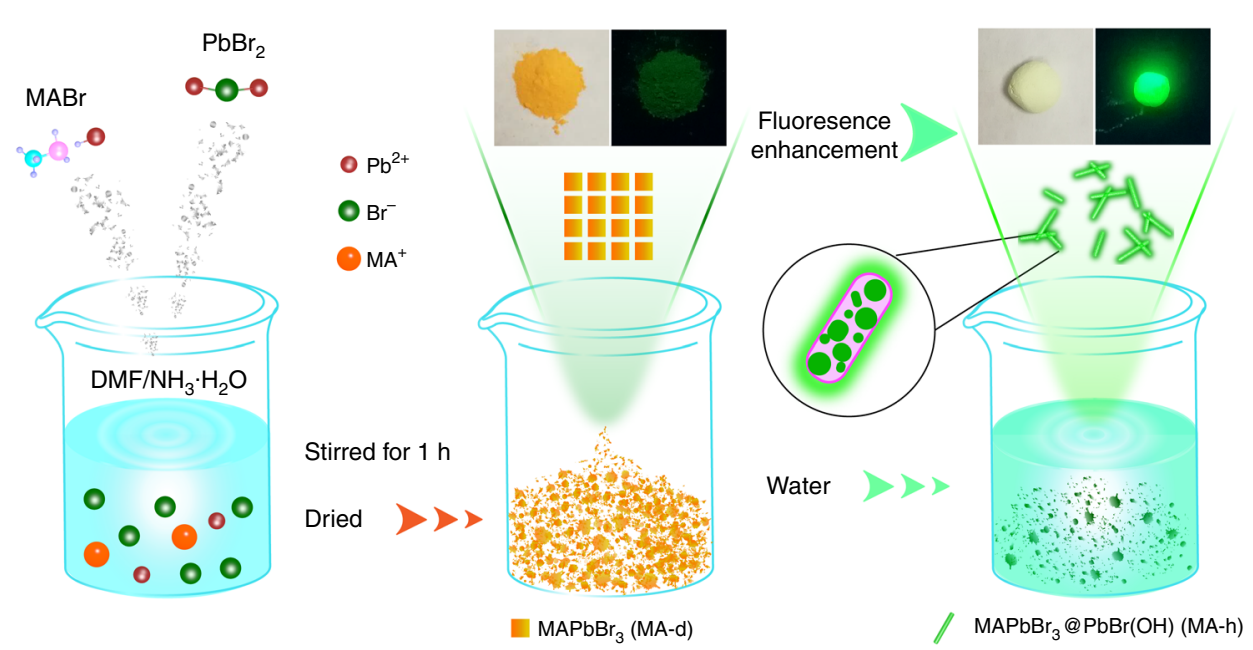

Fig. 1 Schematic diagram of the synthesis process for water-induced $\mathrm{MAPbBr}_{3} @ \mathrm{PbBr}(\mathrm{OH})$

nanocrystals by applying a seeded growth approach, which provided improved PL QY. Wang et al. produced a $\mathrm{Rb}_{4} \mathrm{PbBr}_{6}$ shell to protect $\mathrm{CsPbX}$ through the rubidium oleate posttreatment method. Similarly, Tang et al. reported a kind of $\mathrm{CsPbBr}_{3} / \mathrm{CdS}$ core/shell structure using a hot-injection method. Embedding $\mathrm{CsPbBr}_{3}$ into $\mathrm{SiO}_{2}$ or the pores of mesoporous silica can also improve the stability of the perovskite ${ }^{33-37}$. Although most inorganic matrices are dense and thermally stable, it is difficult to controllably form an inorganic protective layer covering perovskite quantum dots (QDs) due to the high synthetic temperature and relatively complicated synthesis conditions. Furthermore, the introduction of expensive or toxic elements further hinders the applications of perovskites. Moreover, the phase separation between LHPs and protection media still remains, resulting in size variation, low loading, broad spectra, and low PL QY. Some researchers have demonstrated the positive role of water in the synthesis of LHPs ${ }^{38-40}$. For example, Andrey et al. synthesized stable $\mathrm{CsPbBr}_{3}$ nanocrystals by introducing a suitable amount of water into the reaction mixture ${ }^{41}$. It is important to note that when a large amount of water was introduced in this system, the perovskite still decomposed. Atanu and Kwang demonstrated the aqueous synthesis of various hybrids and all-inorganic halide perovskites in acidic and basic media ${ }^{42}$. The perovskites were stable in water for more than 6 months, but the PL QY of the as-prepared methylamino lead bromide perovskites $\left(\mathrm{MAPbBr}_{3}\right)$ was only $11.7 \%$. These observations indicate that there exists a solubility equilibrium between the crystallization of perovskite and its saturated ionic components in water. Thus, the luminescence and stability of LHPs may be enhanced by water by choosing appropriate $\mathrm{pH}$, metal halide salts, and different organic components.
In this work, we show that the PL QY and stability of LHPs can be greatly enhanced by adding water, and the PL QY of the LHPs can be increased from $2.50 \%$ to $71.54 \%$, while that of a sample in aqueous solution decreases minimally after 1 year. This strategy is universal to $\mathrm{MAPbBr}_{3}$, formamidine lead bromide perovskites $\left(\mathrm{FAPbBr}_{3}\right)$, and $\mathrm{CsPbBr}_{3}$. UV-pumped LEDs have been fabricated by using the prepared perovskites as phosphors, and blemishes including scratches, dust, and fingerprints on the surface of glass can be observed clearly under the illumination of the LEDs, indicating that the LEDs are suitable for manual defect detection.

\section{Results}

\section{Structural and morphology characterization}

The schematic synthesis process for the $\mathrm{MAPbBr}_{3} @ \mathrm{PbBr}(\mathrm{OH})$ is illustrated in Fig. 1. The $\mathrm{pH}$ value of $\mathrm{N}, \mathrm{N}$-dimethylformamide (DMF) was preadjusted to 9.0 through dropwise addition of ammonium hydroxide; a mixture of $\mathrm{PbBr}_{2}$ and $\mathrm{MABr}$ with a mole ratio of 1.05:1 was dissolved in DMF solvent under continuous stirring until a white precipitate was formed. Subsequently, the precipitate was placed into an oven at $70^{\circ} \mathrm{C}$ to obtain dried $\mathrm{MAPbBr}_{3}$ perovskite, as shown in the middle of Fig. 1. The dried $\mathrm{MAPbBr}_{3}$ perovskite is named MA-d for convenience, and the yellow MA-d exhibits very weak fluorescence under UV illumination. After addition of water, bright green fluorescence appears. Detailed characterizations indicate that MA-d changes to rod-shaped $\mathrm{PbBr}(\mathrm{OH})$, and $\mathrm{MAPbBr}_{3}$ QDs are embedded in situ into the $\mathrm{PbBr}(\mathrm{OH})$ microrods to form $\mathrm{MAPbBr}_{3} @ \mathrm{PbBr}(\mathrm{OH})$. $\mathrm{MAPbBr}_{3} @ \mathrm{PbBr}(\mathrm{OH})$ is named MA-h for convenience. Based on the first-principles calculations, the bandgap of $\mathrm{PbBr}(\mathrm{OH})$ is $\sim 3.1 \mathrm{eV}$, which is close to the bandgap of the material $(3.44 \mathrm{eV})$. The valence band maximum originates 

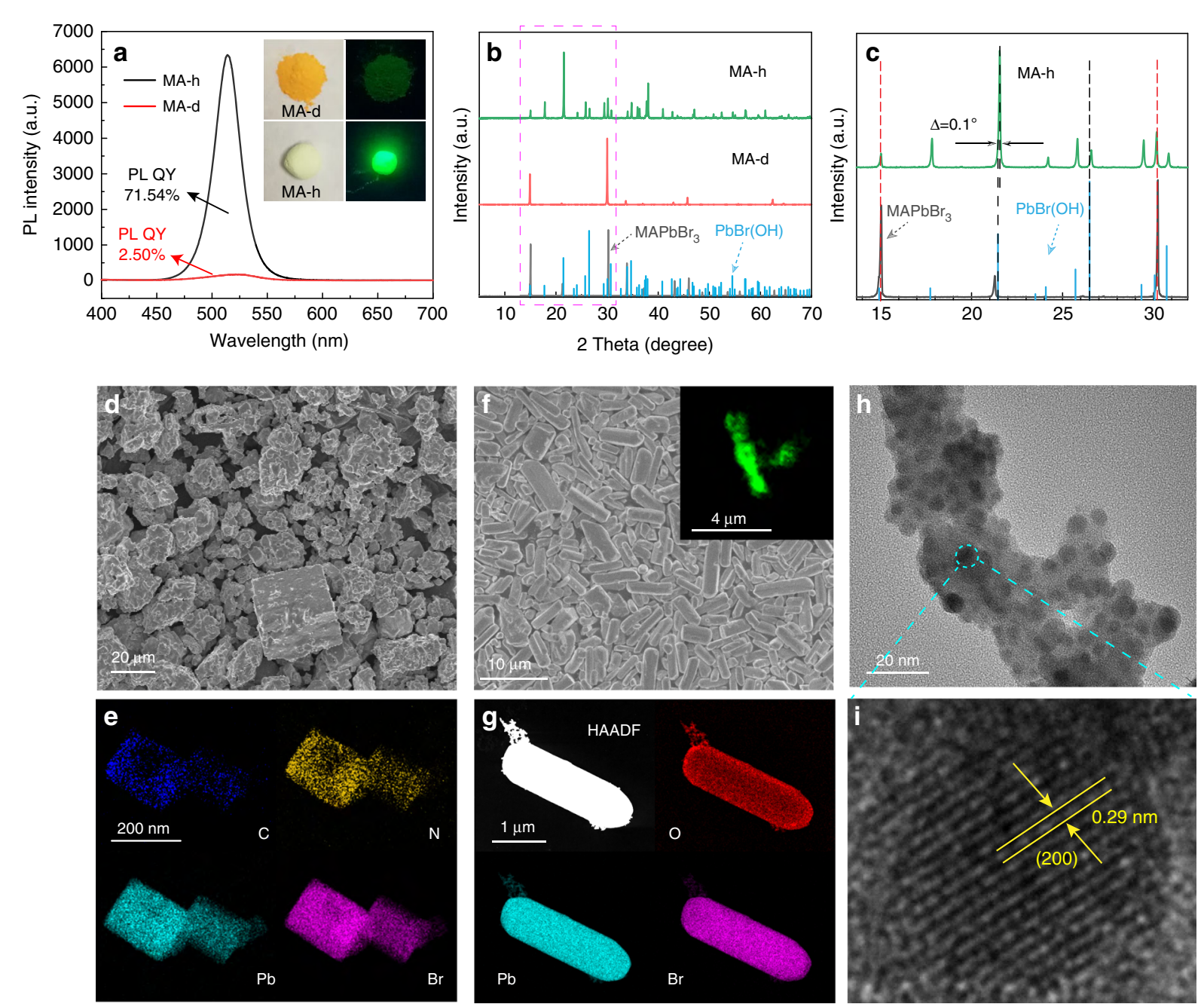

Fig. 2 a PL spectra of MA-d (PL QY: 2.50\%) and MA-h (PL QY: 71.54\%), and the inset shows the corresponding optical images of the two samples under indoor lighting and UV illumination. $\mathbf{b}$ XRD patterns of MA-d and MA-h. $\mathbf{c}$ Magnification of regions marked in $\mathbf{b}$. $\mathbf{d}$ SEM image of MA-d. e Elemental mapping of MA-d. $\mathbf{f}$ SEM image of MA-h, and the inset shows a laser confocal fluorescence microscopy image. $\mathbf{g}$ Elemental mapping of MA-h. $\mathbf{h}$ TEM image of the crushed MA-h. $\mathbf{i}$ HRTEM image of the QDs in MA-h

from the $\mathrm{Br}$ and $\mathrm{O}$ orbitals, and the conduction band $(\mathrm{CB})$ minimum is dominated by the $\mathrm{Pb}$ orbitals, as shown in Supplementary Fig. 1. The decomposition enthalpies of $\mathrm{MAPbBr}_{3}$ and $\mathrm{PbBr}(\mathrm{OH})$ are 0.38 and $16.15 \mathrm{eV}$, respectively, (Supplementary Fig. 2, Supplementary Table 1), indicating that $\mathrm{PbBr}(\mathrm{OH})$ has higher thermodynamic stability than $\mathrm{MAPbBr}_{3}$, which can prevent the decomposition of internal $\mathrm{MAPbBr}_{3}$ QDs. Different from that apt to degrade and lose fluorescence in the presence of moisture $^{43}$, the MA-h synthesized in this work can maintain its bright fluorescence for a year in water.

The MA-d powders are yellow in color and show negligible green fluorescence under UV illumination. The MA-h powders are a greenish color and show bright green fluorescence under UV illumination, and the corresponding picture is shown in the inset of Fig. 2a. The fluorescence spectra of MA-d and MA-h are shown in Fig. 2a, and the emission intensity of MA-h increases 35-fold compared with that of MA-d. To compare the PL peaks of MA-d and MA-h, their PL intensities were normalized, as shown in Supplementary Fig. 3. The PL peak of MA-d is located at $523 \mathrm{~nm}$, while that of MA-h is located at $514 \mathrm{~nm}$, indicating that the size of MA-d decreases after adding water. In addition, the full-width at half-maximum (FWHM) of MA-d is $51 \mathrm{~nm}$, and the FWHM of MA-h is only $26 \mathrm{~nm}$, indicating the smaller and uniform size distribution of MA-h. The crystal structures of MA-d and MA-h were characterized by X-ray diffraction (XRD), as shown in Fig. 2b. All the diffraction peaks of MA-d are from the cubic perovskite structure of 
$\mathrm{MAPbBr}_{3}$, while the spectrum of MA-h contains many extra peaks in addition to those of $\mathrm{MAPbBr}_{3}$, and the extra peaks can be assigned to $\mathrm{PbBr}(\mathrm{OH})$ (JCPDS No. 89-2492), indicating the formation of $\operatorname{PbBr}(\mathrm{OH})$. The enlarged XRD pattern (Fig. 2c) reveals that the diffraction peaks slightly shift to higher angles by $\sim 0.1^{\circ}$ compared with the standard data of $\operatorname{PbBr}(\mathrm{OH})$, which might be a result of the lattice mismatch between $\mathrm{PbBr}(\mathrm{OH})$ and $\mathrm{MAPbBr}_{3}$. To further investigate the structural evolution of the samples before and after adding water, scanning electron microscopy (SEM) images and transmission electron microscopy (TEM) images were taken. Before adding water, MA-d is a typical cubic structure (Fig. 2d), which matches well with its XRD results. To further explore the microscopic morphology of the samples, the MA-d powders were broken by an ultrasonic cell crusher in toluene. In the TEM image of the crushed MA-d (Supplementary Fig. 4), monodisperse and uniform QDs (10 $\mathrm{nm}$ in size) with cubic shapes can be observed, and the QDs tend to agglomerate on the TEM grids, as previously reported $^{44}$. The inset of Supplementary Fig. 4 highlights that MA-d possesses a well-defined crystalline structure with a characteristic lattice distance of $0.58 \mathrm{~nm}$, corresponding to the d-spacing of the (100) crystal planes of $\mathrm{MAPbBr}_{3} . \mathrm{C}, \mathrm{N}, \mathrm{Pb}$, and $\mathrm{Br}$ can be observed from the elemental mapping of MA-d (Fig. 2e); the elements of $\mathrm{C}$ and $\mathrm{N}$ come from methylamine, while the elements of $\mathrm{Pb}$ and $\mathrm{Br}$ stem from the $\mathrm{PbBr}_{6}$ octahedron. MA-h exhibits uniform rod morphology with an average diameter of $1.5 \mu \mathrm{m}$ and length of $4 \mu \mathrm{m}$, as shown in Fig. 2f and Supplementary Fig. 5. The inset of Fig. $2 \mathrm{f}$ shows the corresponding laser confocal fluorescence microscope image, and uniform green fluorescence can be observed along the rod structure. Elemental mapping of MA-h is shown in Fig. $2 \mathrm{~g}$, and the elements $\mathrm{O}, \mathrm{Pb}$, and $\mathrm{Br}$ are uniformly distributed along the rod structure, which indicates the formation of $\mathrm{PbBr}(\mathrm{OH})$. To explore the inner structure of the rod, the MA-h powders were broken in a cell crusher. Some spherical QDs with an outer shell can be observed (Fig. 2h), and the lattice spacing of the QDs is $0.29 \mathrm{~nm}$, corresponding to the d-spacing of the (200) crystal planes of $\mathrm{MAPbBr}_{3}$ (Fig. 2i). The above results confirm that the $\mathrm{MAPbBr}_{3}$ QDs were coated by $\mathrm{PbBr}(\mathrm{OH})$. Thus, one can conclude that the bulk cubic shape of $\mathrm{MAPbBr}_{3}$ changes to a rod-like shape through the addition of water, and the $\mathrm{MAPbBr}_{3}$ QDs are embedded into $\mathrm{PbBr}(\mathrm{OH})$. In addition, $\mathrm{X}$-ray photoelectron spectroscopy (XPS) spectra were collected to detect the surface chemistry of MA-d and MA-h, as shown in Supplementary Fig. 6. The $\mathrm{Br} / \mathrm{Pb}$ atomic ratios calculated from the XPS spectra are summarized in Supplementary Table 2. From the table, one can see that the $\mathrm{Br} / \mathrm{Pb}$ atomic ratios of MA-d and MA-h are 3.06 and 1.11 , respectively, confirming that these materials are $\mathrm{MAPbBr}_{3}$ and $\mathrm{PbBr}(\mathrm{OH})$.

\section{Discussion}

\section{Photophysical property}

To evaluate the stability of the as-prepared perovskites, a repetitive hydrate-dehydrate cycle test was carried out, and the change in the PL intensity was monitored, as shown in Fig. 3a. The PL intensity of the MA-d perovskite increases instantly with the addition of water, accompanied by a color change from yellow to greenish. The perovskite gradually turns back to a yellow color after drying at $60^{\circ} \mathrm{C}$. As the hydrating-dehydrating cycle repeats, the PL intensity of MA-h decreases, while the color of the sample gradually stabilizes at greenish under ambient conditions. Additionally, the PL intensity of MA$\mathrm{d}$ increases after several hydrating-dehydrating cycles, with its yellow color changing to a greenish color. The corresponding evolution of the PL spectra and the color of both samples are shown in Supplementary Figs. 7 and 8. In the first round, the PL QY of MA-d is only $2.5 \%$ and increases to $71.54 \%$ after the addition of water. The sample is held in water for 1 year, and the PL QY of the MA-h is still at $89.9 \%$ of its initial value, i.e., $64.28 \%$, which is one of the most stable fluorescence perovskite powders ever reported to the best of our knowledge. A slight hypsochromic shift of PL is observed during the hydrating-dehydrating cycles, as evidenced by the normalized PL spectra shown in Fig. 3b. The continuous hypsochromic shift in the PL spectra might be a consequence of the decrease in the perovskite QD size during cycling. In addition, traditional LHPs are highly sensitive to polar solvents due to their inherent ionic crystal structure, and the optical properties and even structural integrity of these materials usually deteriorate in polar organic solvents ${ }^{30}$. However, in our work, the MA-h powders show ultrastability to many common organic solvents, such as DMSO, dimethyl formamide, ethanol, acetone, ethylacetate, and benzene. The PL spectra of the MA-h powders immersed in these organic solvents are shown in Fig. 3c, d, and the corresponding images under UV illumination are shown in the insets of Fig. 3c, d. The emission of the MA-h powders is stable in the different organic solvents, and no obvious emission peak shift is observed. In Supplementary Fig. 9, no obvious structural or morphological changes are observed in DMF or DMSO, indicating that $\mathrm{PbBr}(\mathrm{OH})$ is almost insoluble in common solvents, which is why MA-h can maintain good stability. Thermal stability is another important indicator for luminescent materials ${ }^{45}$, especially for LHPs. Thermoinduced particle regrowth would induce PL quenching, and the crystal structure of the perovskites would collapse directly under exceedingly high temperatures. Additionally, high temperature might accelerate the rates of oxidation and hydration, which means that the oxygen- and moisture-induced decomposition would be amplified, leading to more rapid PL quenching ${ }^{30}$. Fig. 3e shows the 

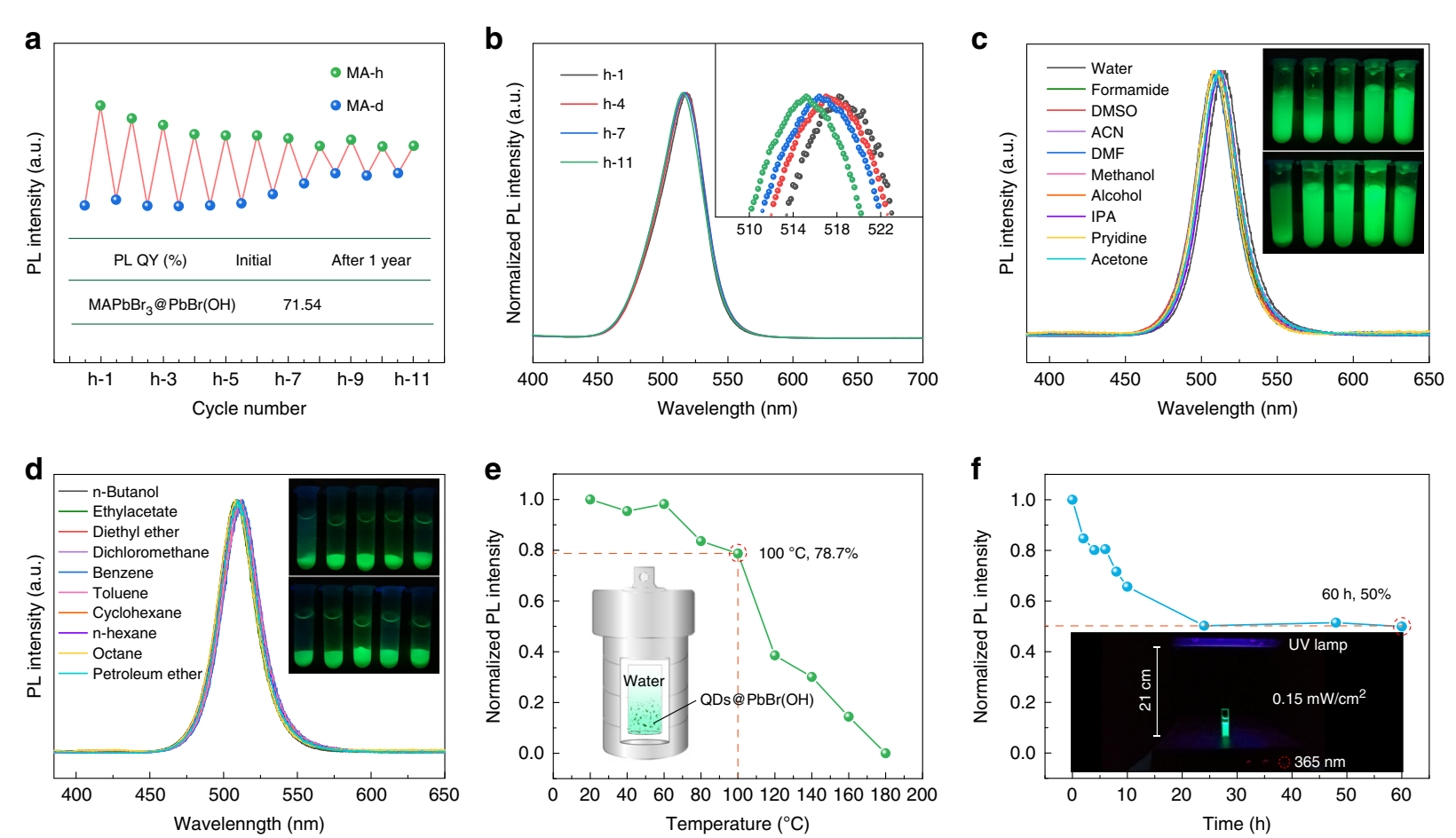

Fig. 3 a The PL intensity of the sample during cycling. $\mathbf{b}$ The corresponding PL spectra of MA-h, and the inset is the magnified spectra. $\mathbf{c}$, $\mathbf{d}$ The PL spectra and images of the MA-h powders immersed in different solvents. e The PL intensity of the sample in the presence of water at different temperatures, and the inset is the schematic diagram of the reaction. $\mathbf{f}$ The PL intensity of the sample in the presence of water under UV irradiation for different hours, and the inset shows the schematic diagram of the test

thermal stability of MA-h in the temperature range from $20^{\circ} \mathrm{C}$ to $180^{\circ} \mathrm{C}$, and the corresponding PL spectra are shown in Supplementary Fig. 10. It is worth noting that the entire test process was carried out in an autoclave filled with water. It can be clearly seen that the PL intensity of MA-h decreases slowly as the temperature increases in the range of $20-100^{\circ} \mathrm{C}$, indicating good thermal stability. Upon heating above $100^{\circ} \mathrm{C}$, the PL intensity decreases sharply and eventually vanishes when the temperature reaches $180^{\circ} \mathrm{C}$. The PL decrease is due to the destruction of dense matrix $\mathrm{PbBr}(\mathrm{OH})$, and the corresponding XRD and SEM images are shown in Supplementary Fig. 11. In addition, the PL intensity of MA-h is still at $78.7 \%$ of the initial intensity upon heating from $20^{\circ} \mathrm{C}$ to $100^{\circ} \mathrm{C}$. The photostability of MA-h was also investigated. Figure $3 \mathrm{f}$ shows the PL spectra of MA-h under continuous irradiation with a UV lamp $(365 \mathrm{~nm}$, $0.15 \mathrm{~mW} / \mathrm{cm}^{2}$ ), and the corresponding PL spectra are shown in Supplementary Fig. 12. The intensity decreases sharply during the first $24 \mathrm{~h}$ and maintains $50 \%$ of the initial intensity after $60 \mathrm{~h}$ of UV illumination. Supplementary Fig. 13 shows the XRD patterns and SEM images of MA-h under UV irradiation, and no obvious structural or morphological changes can be observed. According to previous studies, a possible reason for the PL decrease under UV illumination is photoassisted ionization or photoinduced defects ${ }^{46,47}$. The above results indicate that MA-h has good ambient, thermal, and photostability.

\section{Mechanism for enhanced efficiency}

To further investigate the high PL QY and the luminescence mechanisms of the as-prepared perovskite in the presence of water, the steady-state PL spectra, absorption spectra, time-resolved spectra, and temperaturedependent PL spectra of the as-prepared perovskite were recorded, as shown in Fig. 4. The PL, PL excitation (PLE), and absorption spectra of the as-prepared MA-d perovskite are shown in Fig. 4a, and the samples have strong absorption at $516 \mathrm{~nm}$. Figure $4 \mathrm{~b}$ shows the PL, PLE, and absorption spectra of MA-h, from which semblable PL and excitation characteristics can be observed, while obvious differences are observed in the absorption spectra. Compared with MA-h, MA-d has a significant absorption peak at $516 \mathrm{~nm}$ that absorbs the emissions of MA-d. The absorption peak centered at $\sim 310 \mathrm{~nm}$ with an absorption edge of $360 \mathrm{~nm}$ can be ascribed to the absorption of $\mathrm{PbBr}(\mathrm{OH})$. To further understand the luminescence mechanism of MA-h, temperature-dependent PL measurements were carried out with temperatures from $10 \mathrm{~K}$ to $300 \mathrm{~K}$. In Fig. $4 \mathrm{c}$, the PL peak $(523 \mathrm{~nm})$ at $10 \mathrm{~K}$ is as narrow as $6.8 \mathrm{~nm}$, which originates from the emission of a typical strong excitonic 


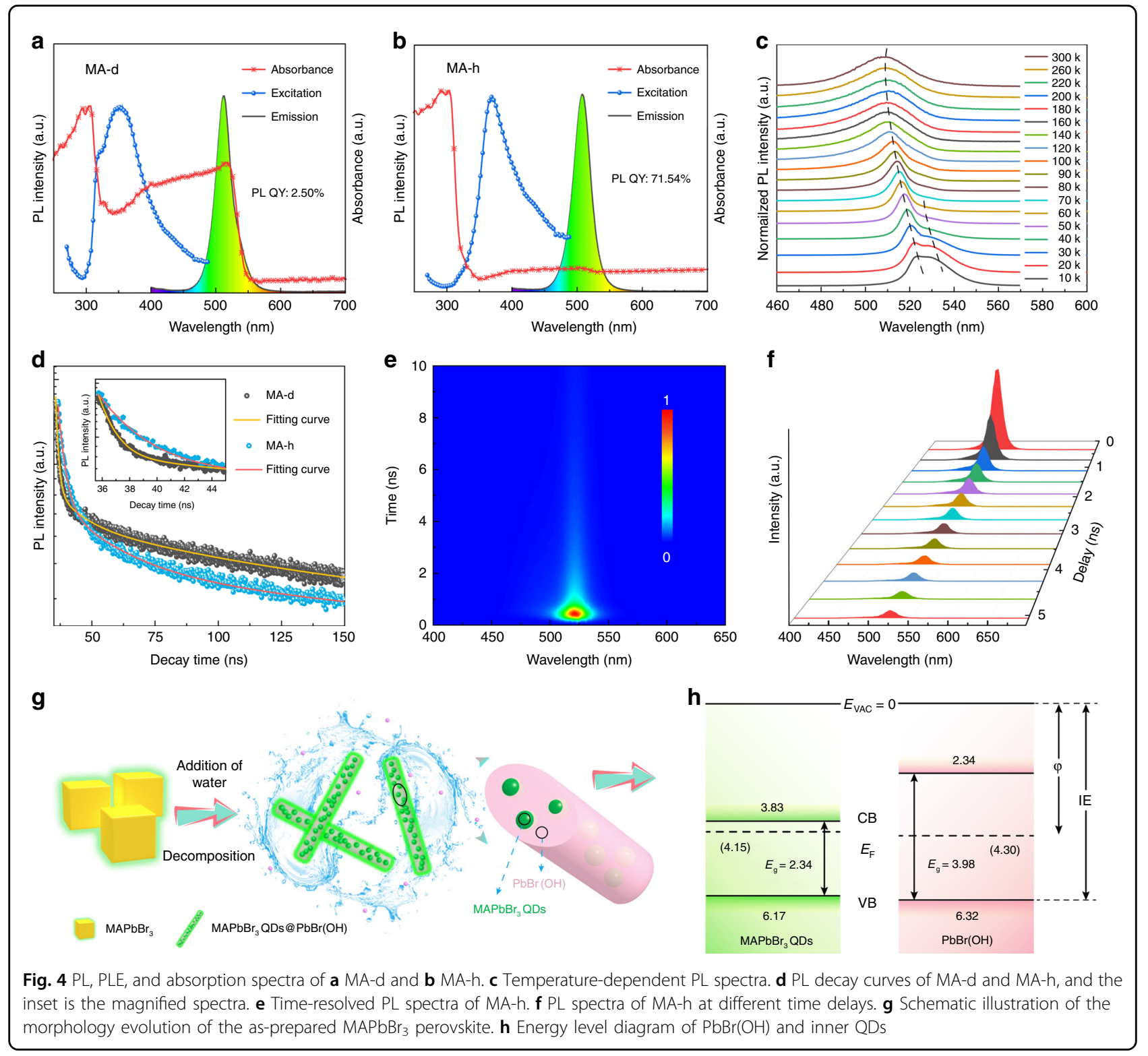

recombination ${ }^{31}$. With the increasing temperature, the intensity of the peak centered at $530 \mathrm{~nm}$ (at $10 \mathrm{~K}$ ) gradually decreases and ultimately disappears at $60 \mathrm{~K}$. Additionally, a new emission peak appears in the shorter wavelength range that corresponds to free exciton emissions. By tracking the PL evolution versus temperature, we clearly reveal that the PL of MA-h at room temperature is dominated by free exciton emissions. The lifetimes of the as-prepared perovskites with and without water were recorded, as shown in Fig. 4d. Both PL decay curves of MA-d and MA-h can be fitted well by a three-exponent function, and the concrete values are summarized in Supplementary Table 3. The average lifetime of MA-h is $2.50 \mathrm{~ns}$, which is longer than that of MA-d (1.08 ns). This result is supported by the time-resolved spectrum of MA$\mathrm{h}$, as shown in Fig. 4e. The longer PL lifetime and higher
PL intensity of MA-h indicate a lower trap-state density ${ }^{48}$, which suggests that $\mathrm{PbBr}(\mathrm{OH})$ effectively passivates the defect sites in $\mathrm{MAPbBr}_{3}$ perovskite. In addition, oxygen molecules can increase the PL efficiency in bulk structures but have the opposite effect in QDs ${ }^{49}$. The instability of halide perovskite caused by ion migration is a possible issue $^{50}$. Ion migrations are usually caused by halide vacancies, and the halide vacancies are passivated by the $\mathrm{Br}$ ions of $\mathrm{PbBr}(\mathrm{OH})$ in this work. Therefore, this strategy can decrease the instability caused by ion migration. Figure $4 \mathrm{f}$ shows the 3D plot of the PL spectra at various times, revealing an essentially time-independent spectral shape of PL emissions. In addition, the FWHM and emission peak are recorded in Supplementary Fig. 14, and the results suggest an absence of peak shifting or broadening. 

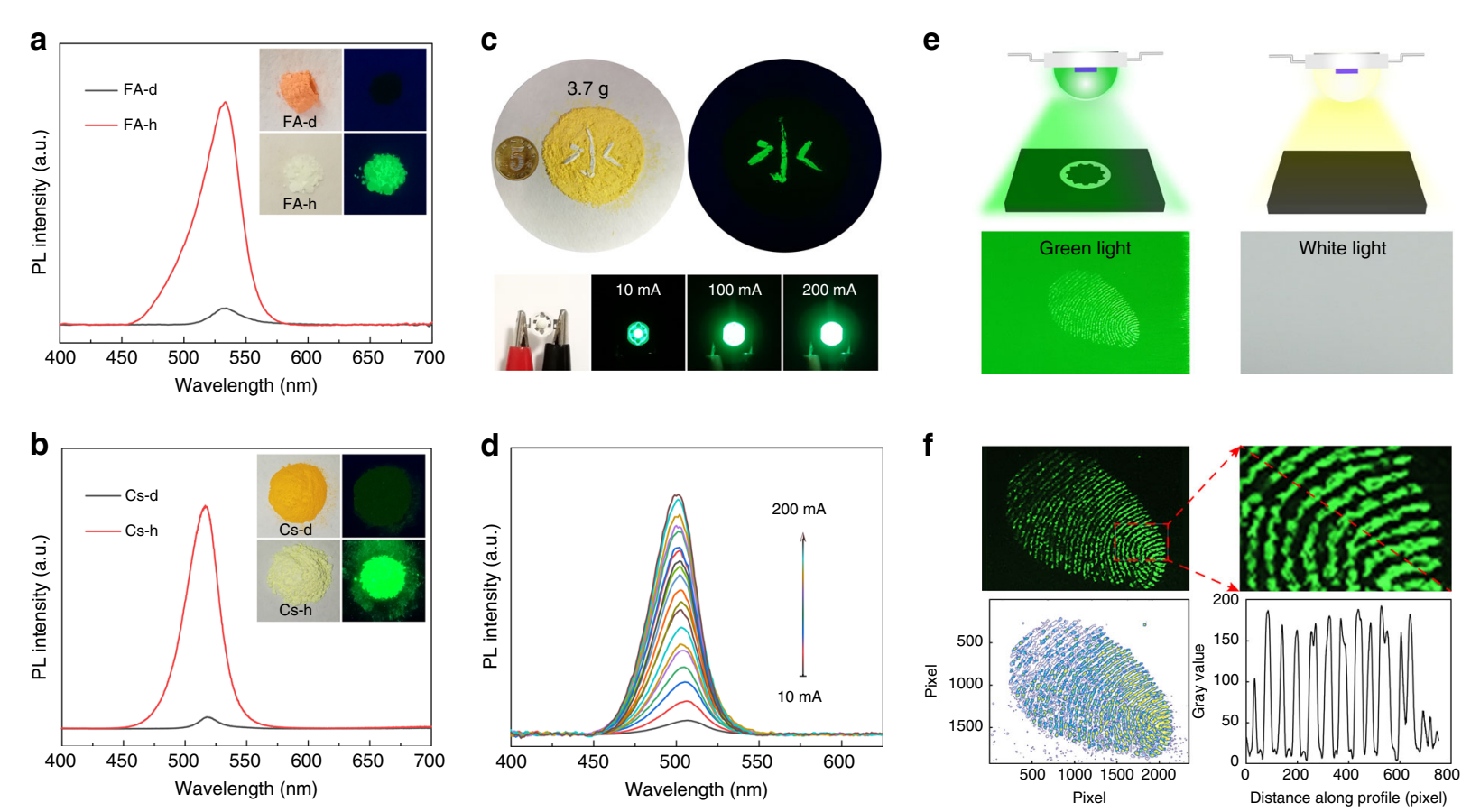

Fig. 5 PL spectra of $\mathbf{a}$ FA lead bromide perovskite and $\mathbf{b}$ all-inorganic halide perovskites, and the insets show digital images of the corresponding samples in ambient light and under UV light. c Digital images of the large-scale synthesized sample (upper images) and LED based on the sample under different driven currents (bottom images). d EL spectra of the LEDs under different driven currents from $10 \mathrm{~mA}$ to $200 \mathrm{~mA}$. e Schematic diagram of potential fingerprint detection based on the as-prepared LED. $\mathbf{f}$ Photographs of fingerprint (upper images) and the corresponding pseudocolor map and gray values along the profile (bottom images)

To further investigate the formation mechanism of MA$h$ in the presence of water, we studied the morphological evolution of MA-h, and a possible mechanism was proposed. The morphological evolution of MA-h during the drying process was observed by optical microscopy and fluorescence microscopy. In Supplementary Fig. 15, the MA-h self-assembles into a cubic structure with loss of water, and then the rod structure forms again after water is added. Figure $4 \mathrm{~g}$ shows a schematic illustration of the formation process of the as-prepared sample in the presence of water. Initially, yellow bulk perovskite with $\mathrm{NH}^{4+}$ and $\mathrm{OH}^{-}$attached to the surface is produced. With the addition of water, the yellow bulk perovskite is destroyed and decomposes from the outside to inside, and $\mathrm{PbBr}$ $(\mathrm{OH})$ is simultaneously formed with the aid of $\mathrm{OH}^{-}$ during this process. A portion of the decomposed small crystals (QDs) are wrapped into rod-shaped $\operatorname{PbBr}(\mathrm{OH})$ by oriented self-assembly. The sample exhibits improved PL QY and ultrahigh stability due to the formation of $\mathrm{MAPbBr}_{3}$ QDs embedded into the $\mathrm{PbBr}(\mathrm{OH})$ matrix. With the volatilization of water during the drying process, $\mathrm{PbBr}(\mathrm{OH})$ reacts with precipitated $\mathrm{MABr}$ (MABr is soluble in water) to form cubic perovskite. Correspondingly, the color of the sample changes from greenish to yellow, and the fluorescence intensity decreases after drying. Subsequently, each cycle (adding water and drying) produces a similar phenomenon until all the $\mathrm{MABr}$ is taken away by water and the perovskite QDs are entirely coated by $\mathrm{PbBr}(\mathrm{OH})$. Therefore, the color of the sample gradually changes to greenish in ambient light, and ultrastable $\mathrm{MAPbBr}_{3} @ \mathrm{PbBr}(\mathrm{OH})$ is formed. The energy level of the $\mathrm{MAPbBr}_{3}$ QDs and $\mathrm{PbBr}(\mathrm{OH})$ was studied by ultraviolet photoelectron spectroscopy (UPS), which can provide both the ionization potential and the valence band (VB) level of the sample, as well as its work function $(\phi)$. By combining UPS (Supplementary Fig. 16) and absorption spectra, we can establish the energy level. In Fig. 4h, the $\mathrm{CB}$ of the matrix $(\mathrm{PbBr}(\mathrm{OH}))$ is higher than that of the QDs, while the VB of the matrix is lower than that of the QDs. As a result, the surface defects are passivated by $\mathrm{PbBr}(\mathrm{OH})$, and both electrons and holes are confined in the QDs. The band alignment between the inner QDs and the outer matrix can guarantee exciton generation and high-rate radiative recombination of the QDs, thus resulting in a sharp PL QY.

\section{Application to LEDs}

Interestingly, this strategy is universal to other bromide perovskites, including $\mathrm{FAPbBr}_{3}$ perovskites and $\mathrm{CsPbBr}$. The PL spectra of $\mathrm{FAPbBr}_{3}$ are shown in Fig. 5a. The corresponding optical properties, structure, and morphology are shown in Supplementary Figs. 17 and 18. 
Table 1 Summary of PL properties and stability of perovskite QDs

\begin{tabular}{|c|c|c|c|c|c|c|}
\hline Strategy & Perovskite & $\begin{array}{l}\text { Emission } \\
\text { peak }(\mathrm{nm})\end{array}$ & FWHM (nm) & PL QYs (\%) & Stability & Ref. \\
\hline \multirow{3}{*}{$\begin{array}{l}\text { Compositional } \\
\text { engineering }\end{array}$} & $\mathrm{CsPbBr}_{3}$ QDs & 513 & 20 & 95 & $90 \%$ (30 d, air) & 31 \\
\hline & $\mathrm{FAPbBr}_{3} \mathrm{NCs}$ & 530 & 22 & 85 & $38 \%\left(100^{\circ} \mathrm{C}\right)$ & 51 \\
\hline & $\mathrm{CsPbBr}_{3}: \mathrm{Mn} \mathrm{QDs}$ & $514-517$ & 20 & 90 & $60 \%$ (120 d, air) & 52 \\
\hline \multirow[t]{3}{*}{ Surface engineering } & $\mathrm{MAPbBr}_{3}$-APTES NCs & 505 & 42 & 55 & $70 \%$ (2.5 h, isopropanol) & 53 \\
\hline & $\mathrm{CsPbBr}_{3}-\mathrm{TDPA}_{\mathrm{QDs}}$ & 522 & 22 & 68 & $80 \%$ (300 min, water) & 54 \\
\hline & $\mathrm{CsPbBr}_{3}-\mathrm{CTAB}$ QDs & 515 & 39 & 71 & $63 \%$ (80 min, UV) & 55 \\
\hline \multirow[t]{6}{*}{ Matrix encapsulation } & $\mathrm{CsPbBr}_{3}-\mathrm{Meso}_{-} \mathrm{SiO}_{2} \mathrm{QDs}$ & 515 & 20 & 55 & $60 \%\left(100^{\circ} \mathrm{C}\right)$ & 33 \\
\hline & $\begin{array}{l}\mathrm{CsPbBr}_{3}-\mathrm{Ami}_{-}-\mathrm{SiO}_{2} \\
\text { powders }\end{array}$ & 520 & 26 & 56 & $80 \%(108 \mathrm{~h}, \mathrm{UV})$ & 47 \\
\hline & $\mathrm{CsPbBr}_{3}-\mathrm{PMMA}$ powders & 510 & 25 & 45 & $75 \%$ (3 d, air) & 56 \\
\hline & $\mathrm{MAPbBr}_{3}$ powders & 518 & 50 & 11.7 & $82 \%$ (6 months, water) & 42 \\
\hline & $\mathrm{Cs} \mathrm{PbBr}_{3}$ powders & 508 & 45 & 53.9 & 74\% (6 months, water) & 42 \\
\hline & $\mathrm{MAPbBr}_{3}$ powders & 514 & 28 & 71.5 & $\begin{array}{l}90 \% \text { (1 year, water, DMF), } 80 \% \\
\left(100^{\circ} \mathrm{C} \text {, water), } 50 \% \text { (60 } \mathrm{h}, \mathrm{UV}\right)\end{array}$ & Our work \\
\hline
\end{tabular}

Figure $5 \mathrm{~b}$ shows the PL spectra of $\mathrm{CsPbBr}_{3}$, and the corresponding optical properties, structure, and morphology are shown in Supplementary Figs. 19 and 20. In addition, $\mathrm{MAPb}(\mathrm{Br} / \mathrm{I})_{3} @ \mathrm{~Pb}(\mathrm{Br} / \mathrm{I})(\mathrm{OH})$ was prepared by this method, and the corresponding spectrum and image are shown in Supplementary Fig. 21. The fluorescence peak redshifts to $\sim 630 \mathrm{~nm}$, indicating that the emissions can be tuned by changing the $\mathrm{X}$ site anion. Notably, this method is simple and facile, and the samples can be prepared on a large scale by proportionally enlarging the precursors, as shown in Fig. 5c. Three grams of the powders are obtained in one synthesis process, and no obvious differences are observed from batch to batch. A Chinese character was written in the samples using water as ink; the regions with water are greenish in color and show bright green fluorescence under UV illumination. As discussed above, compared to traditional perovskites, synthetic powders have improved ambient, thermal, and photostabilities, and the reported perovskites with improved stability are summarized in Table 1 . High PL QY and stability can be achieved simultaneously for the first time in this work. Based on these merits, this material can be employed as a promising phosphor in LEDs. UVpumped LEDs were fabricated by coating the MA-h and PDMS mixture onto $365 \mathrm{~nm}$ UV chips. Bright green light with color coordinates of $(0.21,0.52)$ can be observed (Supplementary Fig. 22), and the emission intensity of the LEDs increases as the drive current increases (Supplementary Fig. 23). The light output of the LED versus time was measured, as shown in Supplementary Fig. 24. The initial luminous intensity of the LED is $1061 \mathrm{~cd} / \mathrm{m}^{2}$, and the intensity decreases with running time. The luminous intensity of the LED settles at $\sim 400 \mathrm{~cd} / \mathrm{m}^{2}$ after $480 \mathrm{~min}$ $(8 \mathrm{~h})$ of running. The luminescence intensity of the asprepared LEDs can reach $9549 \mathrm{~cd} / \mathrm{m}^{2}$ when the driving current increases to $200 \mathrm{~mA}$. The corresponding EL spectra of the LEDs under different drive currents from $10 \mathrm{~mA}$ to $200 \mathrm{~mA}$ are shown in Fig. 5d. In addition, an LED with bright green light can improve the contrast of illuminated objects due to the sensitivity of human eyes to green light. Thus, the as-prepared LEDs are suitable for manual defect detection. As illustrated in Fig. 5e, the light of the LED shines on the surface of the object; thus, the blemishes on the surface of the object (such as scratches, dust, and fingerprints) can be clearly observed. The different defects on the surface of substrates under illumination with a white LED and the as-prepared LED are shown in Supplementary Figs. 25 and 26. Under irradiation with green light, the fingerprint on the glass is extracted (Fig. 5f), and the fingerprint can be observed clearly. The corresponding pseudocolour map and gray values along the lines in Fig. $5 \mathrm{f}$ (bottom images) further indicates the resolution of the image.

In summary, we synthesized bromide-based perovskites whose PL QY can be increased from $2.5 \%$ to $71.54 \%$ by introducing water and decreases minimally in aqueous solution in 1 year. In addition, the as-synthesized $\mathrm{MAPbBr}_{3} @ \mathrm{PbBr}(\mathrm{OH})$ can maintain their luminescence in many kinds of solvents and also exhibit excellent ambient, thermal, and photostabilities. The enhanced 
stability and $\mathrm{PL}$ QY can be attributed to the waterinduced $\mathrm{MAPbBr}_{3} @ \mathrm{PbBr}(\mathrm{OH}) . \mathrm{PbBr}(\mathrm{OH})$ passivated the defects of the $\mathrm{MAPbBr}_{3}$ QDs and confined carriers within the QDs so that $\mathrm{MAPbBr}_{3} @ \mathrm{PbBr}(\mathrm{OH})$ could reach high emission efficiency; additionally, $\mathrm{PbBr}(\mathrm{OH})$ can prevent the exposure of the QDs to air and moisture, thus increasing the stability. This strategy is universal to $\mathrm{MAPbBr}_{3}, \mathrm{FAPbBr}_{3}$, and $\mathrm{CsPbBr}$. UV chip-pumped LEDs were fabricated by using the sample as promising phosphors, and the luminance intensity of the device was as high as $9549 \mathrm{~cd} / \mathrm{m}^{2}$. Furthermore, blemishes including scratches, dust, and fingerprints on the surface of glass could be observed under the illumination of the LEDs, indicating that they are suitable for manual defect detection. This efficient approach for the synthesis of ultrastable and highly efficient luminescent perovskites will push forward their practical applications.

\section{Materials and methods}

The materials used were methylamine $\left(\mathrm{CH}_{3} \mathrm{NH}_{2} ; 32 \%\right.$ wt/wt aq. soln), hydrobromic acid ( $\mathrm{HBr}, 48 \% \mathrm{wt} / \mathrm{wt}$ aq. soln), lead bromide $\left(\mathrm{PbBr}_{2} ;>98 \%\right.$, Aladdin), ammonium hydroxide $\left(\mathrm{NH}_{3} \cdot \mathrm{H}_{2} \mathrm{O} ; 27 \%\right.$ wt $/$ wt aq. soln), and DMF ( $>99.8 \%$, Aladdin). All the reagents were used directly without further purification.

\section{Synthesis of methylammonium bromide}

Methylammonium bromide $\left(\mathrm{CH}_{3} \mathrm{NH}_{3} \mathrm{Br}\right)$ was prepared by slowly mixing methylamine with $\mathrm{HBr}$ at a $1: 1$ molar ratio under continuous stirring for $2 \mathrm{~h}$ at $0^{\circ} \mathrm{C} . \mathrm{CH}_{3} \mathrm{NH}_{3} \mathrm{Br}$ was then crystallized by removing the solvent from an evaporator. Then, the $\mathrm{CH}_{3} \mathrm{NH}_{3} \mathrm{Br}$ was washed with diethyl ether three times. White powders were obtained by recrystallization with ethanol. Subsequently, the powders were dried in vacuum for $24 \mathrm{~h}$ and stored in a dark and dry environment for further use.

\section{Synthesis of $\mathrm{MAPbBr}_{3}$ perovskites}

The $\mathrm{pH}$ of DMF was preadjusted with ammonium hydroxide, and the $\mathrm{pH}$ was $\sim 9$. Then, $2.31 \mathrm{~g} \mathrm{PbBr}_{2}$ and $0.67 \mathrm{~g} \mathrm{MABr}$ (the molar ratio of $\mathrm{PbBr}_{2}$ to $\mathrm{MABr}$ was 1.05) were dissolved in $6 \mathrm{ml}$ alkaline DMF mixed solution to form a white viscous solution, and then the solution was continuously stirred until a white-colored precipitate was formed. Subsequently, the precipitate was centrifuged at $7500 \mathrm{rpm}$, and the precipitate was placed into an oven at $70{ }^{\circ} \mathrm{C}$ to obtain $\mathrm{MAPbBr} r_{3}$ perovskite powders. $\mathrm{MAPbBr}_{3}$ can be converted to bright emissive $\mathrm{MAPbBr}_{3} @ \mathrm{PbBr}(\mathrm{OH})$ through the addition of water.

\section{Synthesis of $\mathrm{MAPbBr}_{3} @ \mathrm{PbBr}(\mathrm{OH})$}

The synthesis details of $\mathrm{MAPbBr}_{3} @ \mathrm{PbBr}(\mathrm{OH})$ are as follows: $1 \mathrm{~g}$ of the as-prepared $\mathrm{MAPbBr}_{3}$ powder was added to $10 \mathrm{ml}$ of water, and then the mixture was stirred for $1 \mathrm{~min}$. The process was replicated several times until the yellow $\mathrm{MAPbBr} 3$ powder transformed into greenish $\mathrm{MAPbBr}_{3} @ \mathrm{PbBr}(\mathrm{OH})$ powder.

\section{Synthesis of $\mathrm{MAPb}\left(\mathrm{Br} / \mathrm{I}_{3} @ \mathrm{~Pb}(\mathrm{Br} / \mathrm{l})(\mathrm{OH})\right.$}

One gram of the as-prepared $\mathrm{MAPbBr}_{3} @ \mathrm{PbBr}(\mathrm{OH})$ was homogeneously mixed with $\mathrm{KI}$ at a mass ratio of $1: 2$, and then $\mathrm{MAPb}(\mathrm{Br} / \mathrm{I})_{3} @ \mathrm{~Pb}(\mathrm{Br} / \mathrm{I})(\mathrm{OH})$ was obtained after several minutes.

\section{Fabrication of LEDs}

First, the as-prepared MA-h phosphors were uniformly mixed with PDMS. The mixture of MA-h and PDMS was debubbled, and coated onto a $365 \mathrm{~nm}$ UV chip and then cured in an oven at $70^{\circ} \mathrm{C}$ for $1 \mathrm{~h}$ to obtain UVpumped LEDs.

\section{Characterization}

The XRD patterns were obtained by an X'Pert Pro diffractometer. The TEM images were taken by a transmission electron microscope (JEM-2010), and the SEM images were obtained on a JEOL JSM6700F field-emission scanning electron microscope. The Fourier transform infrared (FTIR) spectra of the samples were recorded on a Thermo Scientific Nicolet iS10 FTIR spectrometer. The PL QYs of the samples were measured by an Edinburgh fluorescence spectrometer (FLS980). The X-ray photoelectron spectra of the samples were collected by a Thermo Fisher Scientific ESCALAB 250Xi spectrometer equipped with an $\mathrm{Al} \mathrm{Ka} \mathrm{X}$-ray radiation source, and the XPS binding energy was internally referenced to the $\mathrm{C} 1 \mathrm{~s}$ peak $(\mathrm{BE}=284.8 \mathrm{eV})$. The PL spectra were measured by a Hitachi F-7000 spectrophotometer, and the UV-vis absorption spectra were characterized by a UH4150 spectrophotometer.

\section{Computational methods}

All the first-principle calculations were performed utilizing the plane-wave pseudopotential approach within density functional theory as implemented in the Vienna $\mathrm{Ab}$ initio Simulation Package. The $1 \mathrm{~s}$ for $\mathrm{H}, 2 \mathrm{~s}^{2} 2 \mathrm{p}^{2}$ for $\mathrm{C}$, $2 s^{2} 2 p^{3}$ for $\mathrm{N}, 2 \mathrm{~s}^{2} 2 \mathrm{p}^{4}$ for $\mathrm{O}, 6 \mathrm{~s}^{2} 6 \mathrm{p}^{2}$ for $\mathrm{Pb}$, and $4 \mathrm{~s}^{2} 4 \mathrm{p}^{5}$ for $\mathrm{Br}$ were treated as valence electrons, and the interactions between nucleus and valence electrons were described by the projected augmented wave method. The electronic wave functions were expanded in plane-wave basis sets with a kinetic energy of $400 \mathrm{eV}$, and Brillouin zone sample meshes with $2 \pi \times 0.02 \AA^{-1}$ were utilized to ensure the energy convergence of our calculations.

\section{Acknowledgements}

This work was supported by the National Natural Science Foundation of China (grant nos. 11904326, 51602288, U1604263, and 11804307), the Key Science and Technology Project of Henan Province (grant no.171100210600), and the 
Project funded by China Postdoctoral Science Foundation (2019TQ0287 and 2019M662510).

\section{Author contributions}

K.-K.L. and Q.L. contributed equally to this work. K.-K.L. conceived the idea; C.-X. S., L.D., and K.-K.L. supervised and coordinated all aspects of this project. Q.L. carried out the synthesis and characterization, and Q.L. and K.-K.L. wrote the paper. Y.-C.L. fabricated the LEDs. L.-Z.S. measured the time-resolved spectra. D.-W.Y., J.-Y.W., W.-B.Z. and X.-Y.W. commented on the paper, and all of the authors discussed the results.

\section{Data availability}

The data that support the findings of this study are available from the corresponding authors upon reasonable request.

\section{Conflict of interest}

The authors declare that they have no conflict of interest.

Supplementary information is available for this paper at https://doi.org/ 10.1038/s41377-020-0283-2

Received: 25 October 2019 Revised: 8 February 2020 Accepted: 4 March 2020

Published online: 17 March 2020

\section{References}

1. Lee, M. M. et al. Efficient hybrid solar cells based on meso-superstructured organometal halide perovskites. Science 338, 643-647 (2012).

2. Burschka, J. et al. Sequential deposition as a route to high-performance perovskite-sensitized solar cells. Nature 499, 316-319 (2013).

3. Liu, M. Z., Johnston, M. B. \& Snaith, H. J. Efficient planar heterojunction perovskite solar cells by vapour deposition. Nature 501, 395-398 (2013).

4. Jeon, N. J. et al. Solvent engineering for high-performance inorganic-organic hybrid perovskite solar cells. Nat. Mater. 13, 897-903 (2014).

5. Hoye, R. L. Z. et al. Enhanced performance in fluorene-free organometal halide perovskite light-emitting diodes using tunable, low electron affinity oxide electron injectors. Adv. Mater. 27, 1414-1419 (2015).

6. Jeon, N. J. et al. Compositional engineering of perovskite materials for highperformance solar cells. Nature 517, 476-480 (2015).

7. Zhu, H. M. et al. Lead halide perovskite nanowire lasers with low lasing thresholds and high quality factors. Nat. Mater. 14, 636-642 (2015).

8. Senanayak, S. P. et al. Understanding charge transport in lead iodide perovskite thin-film field-effect transistors. Sci. Adv. 3, e1601935 (2017).

9. Yang, W. S. et al. High-performance photovoltaic perovskite layers fabricated through intramolecular exchange. Science 348, 1234-1237 (2015).

10. Chao, X. et al. Ultrasensitive broadband phototransistors based on perovskite/ organic-semiconductor vertical heterojunctions. Light.: Sci. Appl. 6, e17023 (2017).

11. Li, J. H. et al. 50-Fold EQE improvement up to $6.27 \%$ of solution-processed allinorganic perovskite $\mathrm{CsPbBr}_{3}$ QLEDs via surface ligand density control. Adv. Mater. 29, 1603885 (2017).

12. Fang, $H$. H. et al. Photoexcitation dynamics in solution-processed formamidinium lead iodide perovskite thin films for solar cell applications. Light. Sci. Appl. 5, e16056 (2016).

13. Zhang, T. Y. et al. Bication lead iodide $2 \mathrm{D}$ perovskite component to stabilize inorganic a-CsPbl 3 perovskite phase for high-efficiency solar cells. Sci. Adv. 3, e1700841 (2017).

14. Zhao, Y. C. et al. Quantification of light-enhanced ionic transport in lead iodide perovskite thin films and its solar cell applications. Light. Sci. Appl. 6, e16243 (2017).

15. Lin, J. et al. Thermochromic halide perovskite solar cells. Nat. Mater. 17 261-267 (2018)

16. Kim, Y. H. et al. Multicolored organic/inorganic hybrid perovskite light-emitting diodes. Adv. Mater. 27, 1248-1254 (2015).

17. Shi, Z. F. et al. High-efficiency and air-stable perovskite quantum dots lightemitting diodes with an all-inorganic heterostructure. Nano Lett. 17, 313-321 (2017).

18. Sun, H. Z. et al. Chemically addressable perovskite nanocrystals for lightemitting applications. Adv. Mater. 29, 1701153 (2017).
19. Cao, Y. et al. Perovskite light-emitting diodes based on spontaneously formed submicrometre-scale structures. Nature 562, 249-253 (2018).

20. Lin, K. B. et al. Perovskite light-emitting diodes with external quantum efficiency exceeding 20 per cent. Nature 562, 245-248 (2018).

21. Ramasamy, P. et al. All-inorganic cesium lead halide perovskite nanocrystals for photodetector applications. Chem. Commun. 52, 2067-2070 (2016).

22. Swarnkar, A. et al. Quantum dot-induced phase stabilization of a-CsPbl 3 perovskite for high-efficiency photovoltaics. Science 354, 92-95 (2016).

23. Kovalenko, M. V., Protesescu, L. \& Bodnarchuk, M. I. Properties and potential optoelectronic applications of lead halide perovskite nanocrystals. Science $\mathbf{3 5 8}$ 745-750 (2017)

24. Wang, Y. et al. Solution-processed low threshold vertical cavity surface emitting lasers from all-inorganic perovskite nanocrystals. Adv. Funct. Mater. 27 1605088 (2017)

25. Wang, Y. et al. All-inorganic colloidal perovskite quantum dots: a new class of lasing materials with favorable characteristics. Adv. Mater. 27, 7101-7108 (2015).

26. Sutton, R. J. et al. Bandgap-tunable cesium lead halide perovskites with high thermal stability for efficient solar cells. Adv. Energy Mater. 6, 1502458 (2016).

27. Chen, W. W. et al. Enhanced stability and tunable photoluminescence in perovskite $\mathrm{CsPbX}_{3} / \mathrm{ZnS}$ quantum dot heterostructure. Small 13, 1604085 (2017).

28. Li, X. M. et al. All inorganic halide perovskites nanosystem: synthesis, structural features, optical properties and optoelectronic applications. Small 13, 1603996 (2017).

29. Wu, C. et al. Improved performance and stability of all-inorganic perovskite light-emitting diodes by antisolvent vapor treatment. Adv. Funct. Mater. 27, 1700338 (2017)

30. Wei, Y., Cheng, Z. Y. \& Lin, J. An overview on enhancing the stability of lead halide perovskite quantum dots and their applications in phosphor-converted LEDs. Chem. Soc. Rev. 48, 310-350 (2019).

31. Li, X. M. et al. CsPbX 3 quantum dots for lighting and displays: roomtemperature synthesis, photoluminescence superiorities, underlying origins and white light-emitting diodes. Adv. Funct. Mater. 26, 2435-2445 (2016).

32. Tan, Y. S. et al. Highly luminescent and stable perovskite nanocrystals with octylphosphonic acid as a ligand for efficient light-emitting diodes. ACS Appl. Mater. Interfaces 10, 3784-3792 (2018).

33. Wang, H. C. et al. Mesoporous silica particles integrated with all-inorganic $\mathrm{CsPBr}_{3}$ perovskite quantum-dot nanocomposites (MP-PQDs) with high stability and wide color gamut used for backlight display. Angew. Chem. Int. Ed. 55, 7924-7929 (2016)

34. Jia, C. et al. $\mathrm{CsPbX}_{3} / \mathrm{Cs}_{4} \mathrm{PbX}_{6}$ core/shell perovskite nanocrystals. Chem. Commun. 54, 6300-6303 (2018).

35. Tang, X. S. et al. $\mathrm{CsPBBr}_{3} / \mathrm{CdS}$ core/shell structure quantum dots for inverted light-emitting diodes application. Front. Chem. 7, 499 (2019).

36. Zhong, Q. X. et al. One-pot synthesis of highly stable $\mathrm{CsPbBr}_{3} @ \mathrm{SiO}_{2}$ core-shell nanoparticles. ACS Nano 12, 8579-8587 (2018).

37. Wang, B. et al. Postsynthesis phase transformation for $\mathrm{CsPbBr} / \mathrm{Rb}_{4} \mathrm{PbBr}_{6}$ core/ shell nanocrystals with exceptional photostability. ACS Appl. Mater. Interfaces 10, 23303-23310 (2018).

38. Shoyama, $\mathrm{K}$. et al. Effects of water on the forward and backward conversions of lead(ii) iodide to methylammonium lead perovskite. J. Mater. Chem. A 5, 23815-23821 (2017).

39. $\mathrm{Wu}, \mathrm{L}$. Z. et al. From nonluminescent $\mathrm{Cs}_{4} \mathrm{PbX}_{6}(X=\mathrm{Cl}, \mathrm{Br}, \mathrm{I})$ nanocrystals to highly luminescent $\mathrm{CsPbX}_{3}$ nanocrystals: water-triggered transformation through a CsX-stripping mechanism. Nano Lett. 17, 5799-5804 (2017).

40. Turedi, B. et al. Water-induced dimensionality reduction in metal-halide perovskites. J. Phys. Chem. C 122, 14128-14134 (2018).

41. Zhang, X. Y. et al. Water-assisted size and shape control of $\mathrm{Cs} \mathrm{PbBr}_{3}$ perovskite nanocrystals. Angew. Chem. Int. Ed. 57, 3337-3342 (2018)

42. Jana, A. \& Kim, K. S. Water-stable, fluorescent organic-inorganic hybrid and fully inorganic perovskites. ACS Energy Lett. 3, 2120-2126 (2018).

43. Huang, $\mathrm{H}$. et al. Lead halide perovskite nanocrystals in the research spotlight: stability and defect tolerance. ACS Energy Lett. 2, 2071-2083 (2017).

44. Chen, L. C. et al. An ultrasonic synthesis method for high-luminance perovskite quantum dots. Ceram. Int. 43, 16032-16035 (2017).

45. Liang, Y. C. et al. Multi-zinc oxide-cores@uni-barium sulfate-shell with improved photo-, thermal-, and ambient-stability: non-equilibrium sorption fabrication and light-emitting diodes application. J. Colloid Interface Sci. 529 1-10 (2018). 
46. Park, Y. S. et al. Room temperature single-photon emission from individual perovskite quantum dots. ACS Nano 9, 10386-10393 (2015).

47. Li, X. M. et al. Amino-mediated anchoring perovskite quantum dots for stable and low-threshold random lasing. Adv. Mater. 29, 1701185 (2017).

48. Lee, S. et al. Amine-based passivating materials for enhanced optical properties and performance of organic-inorganic perovskites in light-emitting diodes. J. Phys. Chem. Lett. 8, 1784-1792 (2017).

49. Wang, $Y$. et al. Switching excitonic recombination and carrier trapping in cesium lead halide perovskites by air. Commun. Phys. 1, 96 (2018).

50. Yang, D. W. et al. Fast diffusion of native defects and impurities in perovskite solar cell material $\mathrm{CH}_{3} \mathrm{NH}_{3} \mathrm{~Pb}_{3}$. Chem. Mater. 28, 4349-4357 (2016).

51. Minh, D. N. et al. Room-temperature synthesis of widely tunable formamidinium lead halide perovskite nanocrystals. Chem. Mater. 29, 5713-5719 (2017).
52. Zou, S. H. et al. Stabilizing cesium lead halide perovskite lattice through $\mathrm{Mn}(\mathrm{II})$ substitution for air-stable light-emitting diodes. J. Am. Chem. Soc. 139, 11443-11450 (2017).

53. Luo, B. B. et al. Organolead halide perovskite nanocrystals: branched capping ligands control crystal size and stability. Angew. Chem. Int. Ed. 55, 8864-8868 (2016).

54. Xuan, T. T. et al. Highly stable $\mathrm{CsPbBr}_{3}$ quantum dots coated with alkyl phosphate for white light-emitting diodes. Nanoscale 9, 15286-15290 (2017)

55. Cai, Y. T. et al. Improved stability of $\mathrm{CsPbBr}_{3}$ perovskite quantum dots achieved by suppressing interligand proton transfer and applying a polystyrene coating. Nanoscale 10, 21441-21450 (2018).

56. Ma, K. Z. et al. In situ fabrication of halide perovskite nanocrystals embedded in polymer composites via microfluidic spinning microreactors. J. Mater. Chem. C 5, 9398-9404 (2017) 